Editorial

\title{
Near-Infrared light-emitting diodes as a coadjutant therapy for inflammation of cerebral arterial aneurysm
}

\section{Editorial}

Epidemiologically, cerebral arterial aneurysms continue to be a source of mortality/morbidity with all of the improvements of modern technology for diagnosis and therapy. ${ }^{1}$ Recently several articles have been published regarding the association of the arterial wall inflammation size and cerebral arterial aneurysms; the deposition of macrophages is the common denominator. Several medical inflammatory therapies have been suggested, although the potential of change in the growth mechanism could be detrimental for the patient. Histopathologically, the wound healing and inflammatory stages of aneurysms of the endothelium and arterial wall follow the same pattern as others areas of the body. ${ }^{2}$

Near infrared (NIR) light emitting diodes (LED) gallium arsenide (GaAs) were introduced in the 1960 s by NASA for the space agriculture program and wound healing. Over the past 50 years developments have shown significant contributions to healing and decreased inflammation. Experimental applications have shown macrophage deposition significantly decreased with NIR LED GaAs therapy. Decreased macrophages influence levels of growth factors, cytokines, and $\mathrm{CO}^{3}$ Along with these anti-inflammatory properties, NIR LED GaAs also has been shown to increase fibroblasts and collagen, reinforcing arterial walls, allowing for better hemodynamic stability. ${ }^{4}$

This coadjutant therapy can be used in place of anti-inflammatory agents with the potential of increased bleeding risk. This therapy can be influenced by the distance to the aneurysm wall, which varies by techniques. Most of these techniques are non surgical while the patient is preparing for the final invasive therapy. NIR LED GaAs is easy to apply and economical. Animal research trials must be completed before clinical application.

\section{Acknowledgments}

None.
Volume 3 Issue 2 - 2015

\author{
Manuel Dujovny,' Onyekachi Ibe, ${ }^{2}$ Erin \\ Morency ${ }^{3}$ \\ 'Department of Neurosurgery, Wayne State University, Detroit, \\ MI, USA \\ ${ }^{2}$ College of Engineering \& Information Science, DeVry \\ University, Southfield, MI, USA \\ ${ }^{3}$ School of Nursing, Oakland University, Human Health Building, \\ Rochester MI, USA
}

Correspondence: Manuel Dujovny, 196 Long Lake Shore Drive, Bloomfield Hills, Michigan 48386, USA, Tel 248 758-9662, Email manueldujovny@hotmail.com

Received: October 27, 2015 | Published: October 28, 2015

\section{Conflicts of interest}

None.

\section{References}

1. Dujovny M, Agner C, Ibe O, et al. Self-closing aneurysm clip: a historical review. Neurol Res. 2010;32(10):1011-1020.

2. Ollikainen E, Tulamo R, Frösen J, et al. Mast cells, neovascularization, and microhemorrhages are associated with saccular intracranial artery aneurysm wall remodeling. J Neuropathol Exp Neurol. 2014;73(9):855864.

3. Ibe O, Morency E, Sosa P, et al. The role of near-infrared light-emitting diode in aging adults related to inflammation. Healthy Aging Research. 2015;4:24.

4. Turkmani AH, Edwards NJ, Chen PR. The role of inflammation in cerebral aneurysm. Neuroimmunol Neuroinflammation. 2015;2:102106. 